\title{
Estudio probabilístico de métodos avanzados de análisis de curva de declinación para la estimación de reservas de petróleo durante el régimen de flujo transitorio
}

\author{
Daniel Alarcón; Christopher Villafuerte; Víctor Sabrera; Alexei Huerta* \\ Universidad Nacional de Ingeniería \\ *Email: alexeihq@gmail.com
}

\begin{abstract}
Resumen
Los reservorios del Noroeste Peruano han producido por más de un siglo principalmente areniscas silicoclásticas, de baja permeabilidad, a través de yacimientos en compartimentos o sub-bloques generados por un alto fallamiento. El mecanismo característico de impulsión es depletación por gas en solución y los pozos comúnmente son producidos con un sistema de levantamiento artificial por bombeo mecánico o gas lift; el factor de recobro promedio alcanzado en estos yacimientos está entre $8 \%$ y $15 \%$.

En los últimos años se ha observado un gran avance en las técnicas de estimación de reservas a través de análisis de curvas de declinación (DCA) en yacimientos no convencionales de muy baja permeabilidad. Estas técnicas también pueden ser utilizadas en reservorios convencionales apretados, aquellos típicamente encontrados en la Cuenca Talara de Perú, en los cuales la aplicación de la técnica tradicional de DCA suele sobreestimar los pronósticos de producción y el cálculo de reservas. Alternativamente, se ha propuesto evaluar los modelos de Declinación Estrecha Extendida (SEDM), Ley de Potencia, Duong y Declinación Exponencial Extendida, en los reservorios de esta cuenca (Duong, A. N., 2010).

Durante el proceso de evaluación, se utilizó la información de 30 pozos productores de diferentes reservorios apretados de la Cuenca Talara: Manta, Mesa, Mogollón y Pariñas Inferior. Se probaron los citados métodos de análisis de declinación con la historia de producción de los primeros 6, 12 y 24 meses; cabe señalar que, de acuerdo a la observación realizada y debido a la baja productividad de estos reservorios, se determinó que su flujo aún se encontraba en estado transitorio durante el periodo de análisis. Los pronósticos se compararon con datos reales de producción y los modelos de declinación se clasificaron por orden de prioridad de acuerdo a la certeza de su grado de ajuste. Se eligió la técnica más idónea para generar, a través de un enfoque probabilístico, utilizando la simulación Monte Carlo, un conjunto de curvas tipo para la cuenca Talara; de esta manera, se obtuvieron los percentiles P90, P50 y P10.
\end{abstract}

Los rangos de permeabilidad en los reservorios de los casos evaluados varían entre 0.01 y $1 \mathrm{mD}$. Se ha observado que la aplicación del modelo DCA de ARPs en pozos con poca historia de producción de reservorios de baja permeabilidad, resulta impreciso en las predicciones de estimados de reservas. Por otro lado, los nuevos métodos de DCA son más conservadores en sus pronósticos y, por ende, más precisos en este caso debido a que permiten incorporar información en estado transitorio. La aplicación de este nuevo enfoque ayudará a obtener pronósticos confiables en la evaluación de futuras estrategias de desarrollo y a predecir con mayor certeza la producción de futuros trabajos de workover y nuevos pozos de desarrollo.

Palabras clave: Análisis de Curvas de Declinación (DCA); Reservorios de baja permeabilidad; Reservorios convencionales apretados; Reservas; Pronósticos; Monte Carlo; Flujo transitorio; Curva tipo.

Cita: Alarcón, D., Villafuerte, C., Sabrera, V. y Huerta, A. (2020). estudio probabilístico de métodos avanzados de análisis de curva de declinación para la estimación de reservas de petróleo durante el régimen de flujo transitorio. Revista Fuentes: El reventón energético, $18(1), 61-74$. 


\title{
Probabilistic study of advanced methods in decline curve analysis (DCA) used for the estimation of oil reserves during the transient flow regime
}

\begin{abstract}
The reservoirs of the Peruvian Northwest have produced, for more than a century, mainly low permeability silicoclastic sandstones through deposits in compartments or sub-blocks generated by high faulting. The characteristic depletion drive mechanism is solution gas and the wells are commonly built with an artificial lift system by mechanical pumping or gas lift; the average recovery factor reached in these deposits is between $8 \%$ and $15 \%$.

In recent years, a great advance has been observed in reserve estimation techniques through the analysis of decline curves (DCA) in unconventional deposits with very low permeability. These techniques can also be used in tight conventional reservoirs, those typically found in the Talara Basin in Peru, in which the application of the traditional DCA technique often overestimates production forecasts and reserve calculations. Alternatively, it has been proposed to evaluate the Stretched Exponential Decline Model (SEDM), Power Law, Duong and Extended Exponential Decline, in the reservoirs of this basin (Duong, A. N., 2010).

During the evaluation process, information from 30 producing wells from different tight reservoirs in the Talara Basin was used: Manta, Mesa, Mogollón and Pariñas Inferior. The aforementioned decline analysis methods were tested with the production history of the first 6,12 and 24 months; It should be mentioned that, according to the observation made and due to the low productivity of these reservoirs, it was determined that their flow was still in a transitory state during the analysis period. The forecasts were compared with actual production data and the decline models were ranked in order of priority according to the certainty of their degree of adjustment. The most suitable technique was chosen to generate, through a probabilistic approach and using Monte Carlo simulation, a set of type curves for the Talara basin; thus, the P90, P50 and P10 percentiles were obtained.
\end{abstract}

The permeability ranges in the reservoirs in the evaluated cases fluctuate between 0.01 and $1 \mathrm{mD}$. It has been observed that the application of ARPs' traditional DCA model in wells with little production history of low permeability reservoirs, proves to be inefficient in the reserve calculation forecasts. furthermore, the new DCA methods are more conservative in their forecasts and, therefore, more accurate in this case because they allow the incorporation of transient information. The application of this new approach will help to obtain reliable forecasts in the evaluation of future development strategies and to predict with greater certainty the production of future workover jobs and new development wells.

Keywords: Decline curve analysis (DCA); Low permeability reservoirs; Tight conventional reservoirs; Oil forecasts; Monte Carlo Simulation; Transient flow; Type curve.

\section{Introducción}

Uno de los principales objetivos de la ingeniería de petróleo es predecir con certeza la producción futura de un pozo o campo, hay distintas herramientas que pueden utilizarse para alcanzar este propósito, siendo el análisis de curvas de declinación el más sencillo y aceptado dentro de la industria del petróleo. Esta técnica fue propuesta por en 1945 y utiliza datos de producción para encontrar la mejor curva de ajuste histórica, extrapolarla en el futuro y así predecir el comportamiento del campo y calcular sus reservas permeabilidad (Guardia, V. M. D., et al 2011). Sin embargo, esta metodología presenta algunas restricciones, siendo una de ellas que el flujo debe haber alcanzado las fronteras del yacimiento, esto es difícil de lograr en yacimientos apretados debido a que, por su baja permeabilidad, el tiempo en el que se alcanza las fronteras es prolongado. Es así que la aplicación de curvas de declinación convencional en yacimientos apretados con poca historia de producción no es adecuada, ya que el mejor ajuste en los primeros años de producción no refleja el comportamiento futuro (Páez, E. G. Met al 2016).

Con la creciente producción de gas y petróleo proveniente de yacimientos no convencionales, distintos autores han desarrollado nuevas técnicas de DCA que toman en cuenta el flujo en estado transitorio y reflejan en mejor medida el comportamiento de dichos yacimientos de muy baja permeabilidad. Estos métodos son: Método de Declinación Estrecha Extendida ("SEDM" por sus siglas en inglés), Método de Ley de Potencia, Método de Duong y Método de Exponencial Extendido. Es así, que estas nuevas técnicas representan una solución factible para pronosticar la producción y estimar correctamente las reservas de los yacimientos apretados.

\section{Generalidades de la Zona de Estudio}

La Cuenca Talara está ubicada en el noroeste del Perú (ver Figura 1 y 2), donde abarca los departamentos de Piura y Tumbes, en el extremo más occidental de América del 
Sur y perpendicular al cambio de dirección de los Andes peruanos $\mathrm{NO}$ - SE con los Andes Ecuatorianos NE - SO en la deflexión de Huancabamba. Tiene una superficie aproximada de $15,000 \mathrm{~km}^{2}$ de los cuales las $2 / 3$ partes se encuentran costa afuera, una longitud aproximada de $300 \mathrm{~km}$ desde el distrito de Zorritos hasta Islas Lobos de Tierra en Sechura y transversalmente se aproxima a 50 $\mathrm{km}$ desde la cadena montañosa de los Cerros Amotape hasta la zona de subducción de placas.

La cuenca Talara es una depresión estructural terciaria. Los sedimentos han sido depositados con una dirección regional NE-SO y presentan estratos con buzamiento promedio de $25^{\circ}$ al SE. La geología de superficie revela un estilo estructural de complicado fallamiento en bloques que ha sido confirmado en el subsuelo mediante perforaciones efectuadas en la parte continental y el zócalo de la cuenca e indirectamente por levantamientos geofísicos. El fallamiento es del tipo normal; varia de intenso a moderado, con desplazamientos hasta de 8000 pies y ángulos que varían de 30 a $60^{\circ}$ y genera numerosos bloques que presentan buzamientos que varían de 10 a $25^{\circ}$ al $\mathrm{SE}$.

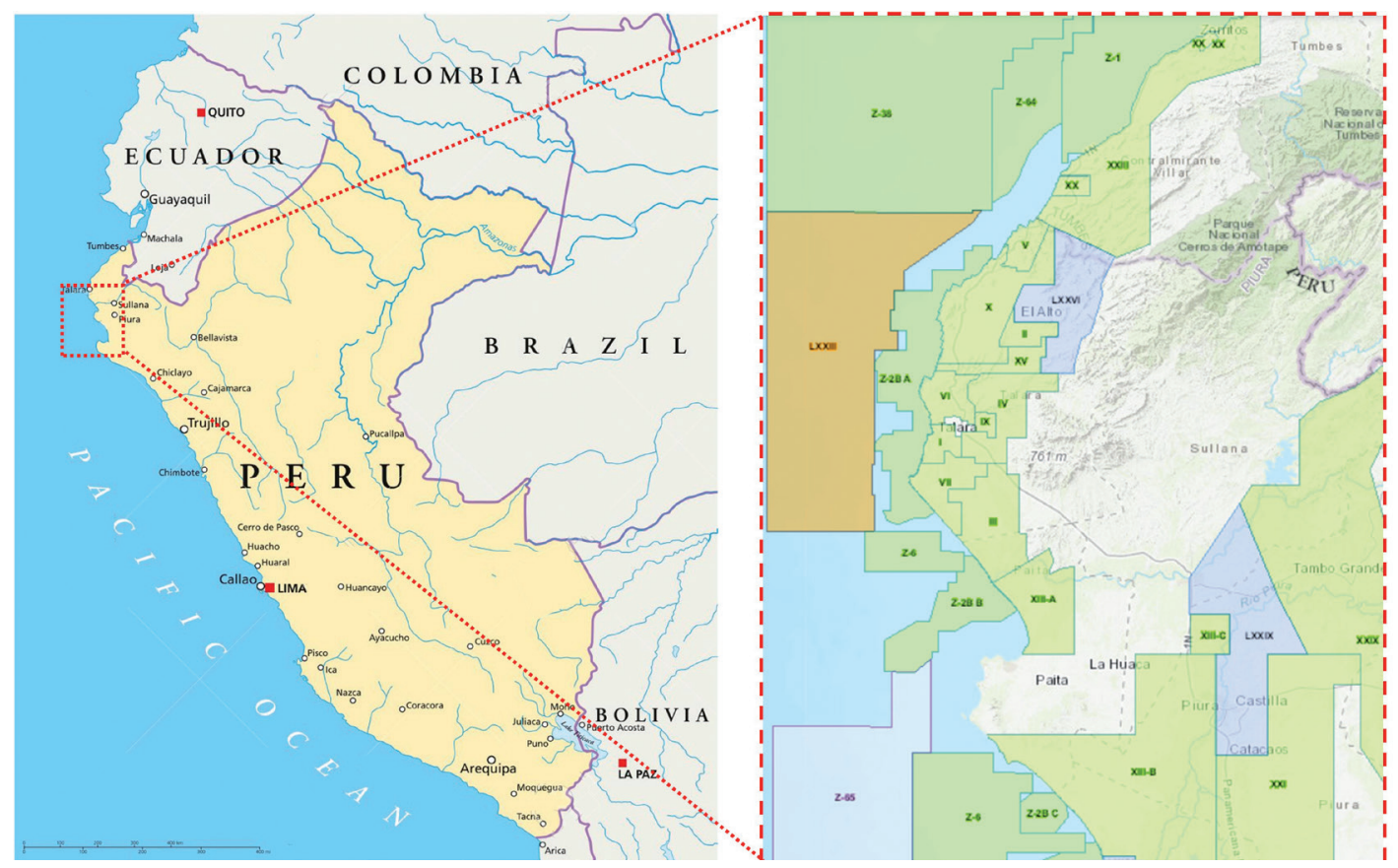

Figura 1. Ubicación geográfica de la cuenca Talara. Fuente: Perupetro.

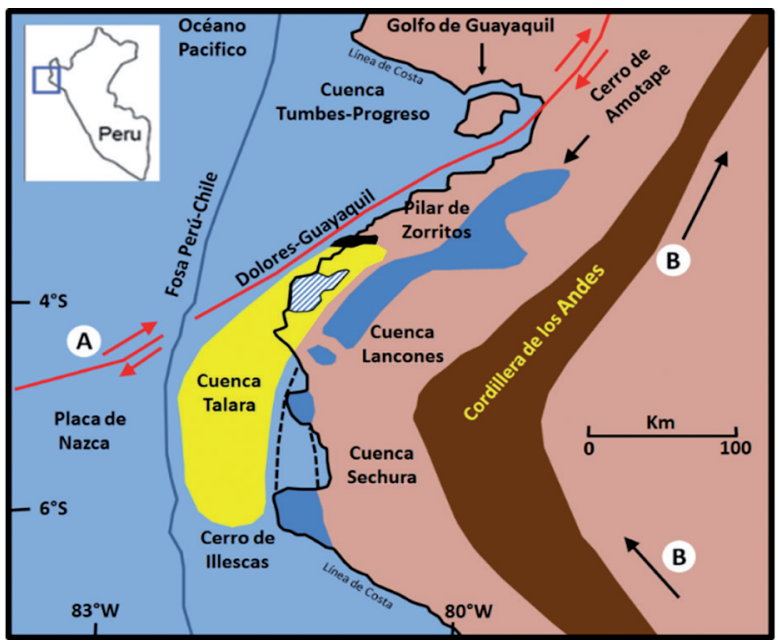

Figura 2. Cuenca Talara - Ubicación Regional. Fuente: Informe de Reservas 2018 del Lote X.

\section{Estratigrafía.}

Desde el punto de vista estratigráfico la cuenca Talara está caracterizada por contener múltiples reservorios desde la edad del Cretácico hasta el Eoceno Superior. La Figura 3 muestra la columna estratigráfica generalizada para la cuenca. En general las rocas madre son las formaciones Muerto y Redondo con múltiples rocas reservorio asociadas en la mayoría de la era terciaria.

\section{Características de Reservorio.}

El mecanismo de empuje predominante en los reservorios de la cuenca Talara es el de empuje por gas en solución, aunque existen zonas con influencia de empuje por capa de gas. El tipo de petróleo promedio en la cuenca por su gravedad específica es clasificado como ligero, entre 30 a $40{ }^{\circ} \mathrm{API}$ (black oil) de bajo encogimiento. Los reservorios poseen en su mayoría 
calidad de reservorio de intermedia a baja (baja porosidad y permeabilidad). En cuanto al nivel de energía, los reservorios presentan gradientes iniciales de presión entre 0.35-0.51 psi/ft, dependiendo de la zona, teniendo en muchos casos pozos surgentes al inicio de etapa productiva de un bloque estructural y el factor de recobro primario final promedio de los reservorios se encuentra entre el $8-15 \%$ del POES (Petróleo Original en sitio).

\section{Características de Pozos.}

Los pozos perforados tienen diversas características (vertical, tipo $\mathrm{S}$, desviado), la completación común es multireservorio (producción en conjunto) y por lo general son estimulados mediante fracturamiento hidráulico por etapas. Posterior a la surgencia los sistemas artificiales típicos utilizados en los pozos son: gas lift y bombeo mecánico, debido a las condiciones y facilidad de captación del gas de producción.

\section{Enunciados teóricos y definiciones}

\section{Método de Arps.}

Presentó el primer modelo de análisis de curvas de declinación, es un análisis empírico basado en un radio de pérdida o radio de declinación " $\mathrm{D}$ " y su derivada " $\mathrm{b}$ ":

$$
\begin{aligned}
& D=-\frac{1}{d} \frac{d q}{d t} \\
& b=\frac{d}{d t}\left(\frac{1}{D}\right)
\end{aligned}
$$

El comportamiento productivo que propone está gobernado por la siguiente ecuación:

$$
q=\frac{q_{i}}{(1+b D t)^{\frac{1}{b}}}
$$

Donde es el caudal incial o caudal máximo del cual se inicia la declinación y "Di" es el radio de pérdida inicial o la declinación inicial mensual de la historia de producción.

La ecuación 3 es la Ecuación Hiperbólica del análisis de curva de declinación, Arps indica que el valor de b debe estar entre 0 y 1 . Cuando este es 0 es debido a que "D" es constante en el tiempo, ya que "b" es la derivada de la inversa de "D", convirtiendo así la ecuación 3 en la ecuación Exponencial:

$$
\mathrm{q}=\mathrm{q}_{\mathrm{i}} * \exp (-\mathrm{Dt})
$$

Este comportamiento se observa en pozos que han alcanzado las fronteras de un yacimiento cerrado.
Y cuando el valor de "b" es igual a 1 la ecuación 3 se simplifica en la Ecuación Armónica mostrada a continuación:

$$
q=\frac{q_{i}}{\left(1+D_{i} t\right)}
$$

Existen distintas metodologías para estimar los valores "b" y "Di", como graficar los valores de " $1 / \mathrm{D}$ " vs tiempo, donde la intersección con el eje es " $1 / \mathrm{Di}$ " y la pendiente es "b", o por otro lado utilizar las curvas tipo presentadas por Fetkovich (Fetkovich, M. J., et al 1996). Sin embargo, estos parámetros pueden ser calculados rápidamente a través de la técnica matemática de regresión múltiple disponible en hojas de cálculo comerciales.

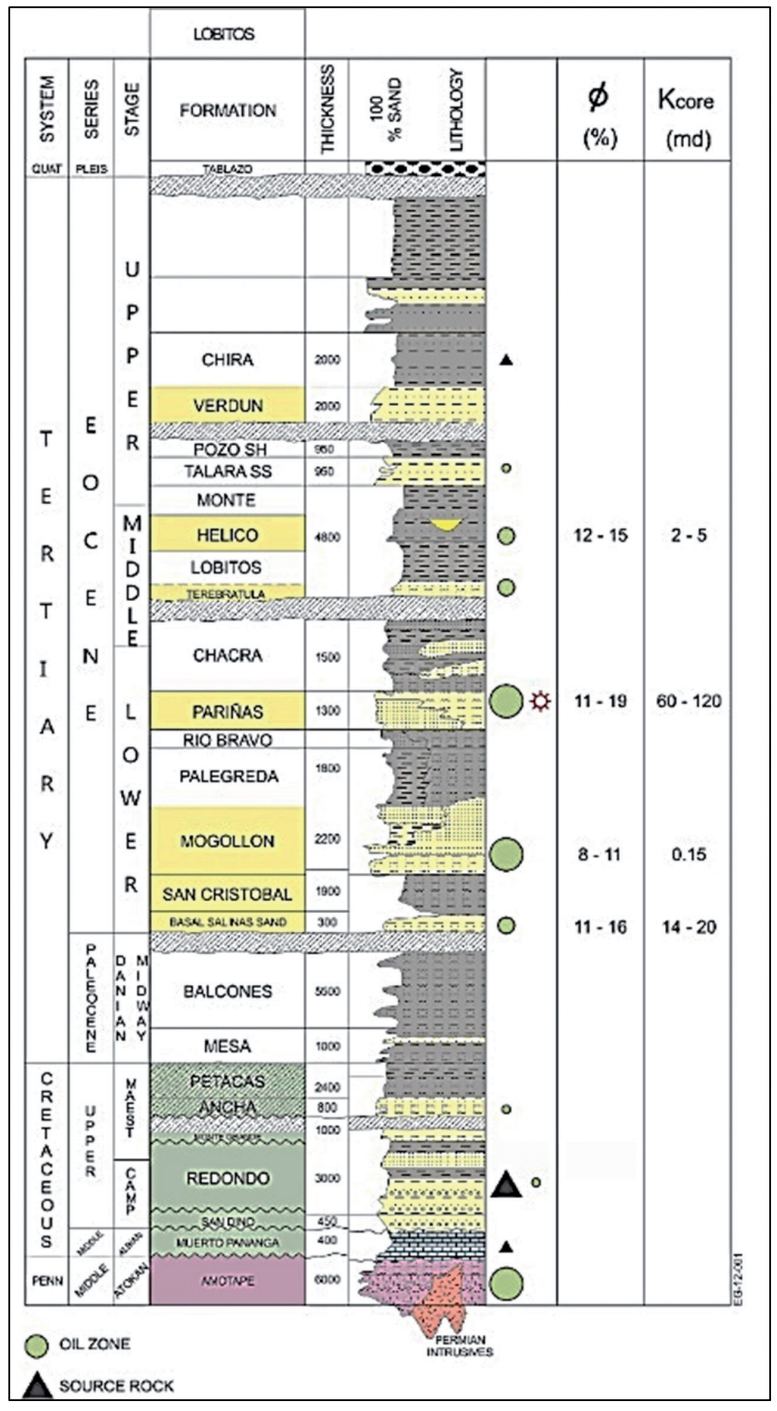

Figura 3. Columna Estratigráfica Generalizada-Cuenca Talara. Fuente: Elaboración Propia - Informe de Reservas 2018 del Lote Z-2B. 
La aplicación de DCA en yacimientos apretados, utilizando información en estado transitorio, no cumple con el rango de b entre 0 y 1 , normalmente se observa valores de ajuste de "b" mayores a 1 lo cual resulta en la sobreestimación de reservas.

En la Figura 4, se muestra el comportamiento típico de las curvas de declinación para yacimientos apretados, se observa que al utilizar información en estado transitorio ninguna de las curvas convencionales se ajustan al comportamiento productivo; sin embargo, cuando se utilizan valores de "b" mayores a 1 se logra el ajuste esperado por un corto tiempo, ya que a medida que se tiene más historia productiva, es común que la producción decline más rápido que la curva ajustada, sobrestimándose así las reservas.

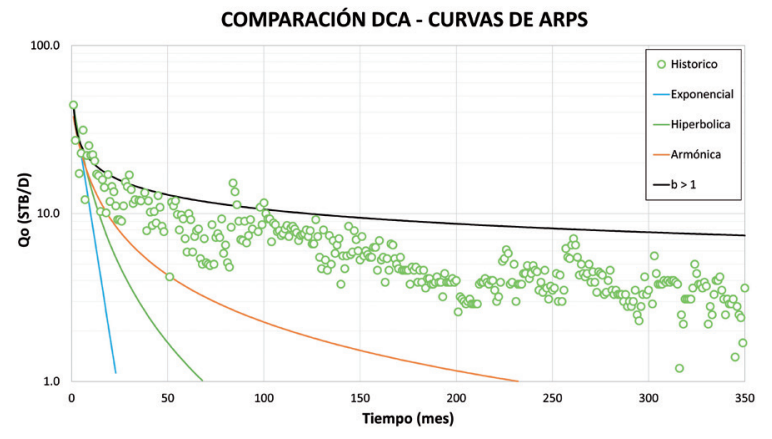

Figura 4. Aplicación de DCA convencional y con "b" mayor a 1, utilizando los 6 primeros meses de producción para el ajuste.

\section{Método de SEDM.}

Valkó presentó un nuevo modelo de análisis de curvas de declinación basado en la evaluación de 10,000 pozos productores de shale gas del campo Barnett en Estados Unidos (Valkó, P. P. 2009).

La curva de declinación presentada es la siguiente:

$$
\mathrm{q}=\mathrm{q}_{\mathrm{i}} * \exp \left(-\left(\frac{\mathrm{t}}{\tau}\right)^{\mathrm{n}}\right)
$$

Dónde t es el tiempo en meses, " $\mathrm{q}_{\mathrm{i}}$ " es el caudal máximo o el inicial de donde empieza la declinación, "n" es un parámetro de declinación adimensional y " $\tau$ " es un parámetro de declinación en meses.

Los dos parámetros " $\tau$ " y " $\mathrm{n}$ " pueden verse como factores de forma y escala que controlan la forma y la magnitud de la tasa de declinación. Estos pueden determinarse con regresión múltiple.

\section{Método de Ley de Potencia.}

Ilk y otros autores presentaron un método de estimación de reservas a través de una curva de declinación "no hiperbólica" (Ilk, D., et al 2008), (a diferencia de la ecuación propuesta por Arps, b no es constante en el tiempo), parte de la ecuación exponencial de Arps cambiando el parámetro D por la ecuación 7 (Arps, J. J. 1945).

$$
\begin{gathered}
D=D_{\infty}+D_{1} t^{-(1-n)} \\
q=\widehat{q}_{1} \exp \left(-D_{\infty} t-\frac{D_{1}}{n} t^{n}\right) \\
q=\widehat{q}_{1} \exp \left(-D_{\infty} t-\hat{D}_{1} t^{n}\right)
\end{gathered}
$$

Donde t es el tiempo de producción en días, “ es el caudal "intercepto", este es el caudal al tiempo 0, cabe resaltar que no es el caudal promedio mensual inicial que se utiliza en la ecuación de Arps (ecuación 3), es la constante de declinación "intercepto" a una unidad de tiempo ("D" inicial o "D" a 1 día de producción), es la constante de declinación a un tiempo infinito, " $n$ " es el exponente del tiempo y es la constante de declinación, que es igual a " $/ \mathrm{n} "$.

El parámetro de radio de declinación " $\mathrm{D}$ " depende de dos factores, por un lado, a cortos tiempos de producción de una ley de potencia en función del tiempo que es un indicador del comportamiento de producción en estado transitorio y otro a tiempos largos que representa el comportamiento de producción dominado por fronteras que es una constante. De la ecuación 7 se puede observar que a medida que avanza el tiempo el valor de disminuye, debido a que $\mathrm{n}$ es menor a 1 , y por otro lado incrementa de valor, viéndose así la relación entre ambos parámetros de declinación con el tiempo de producción del pozo y el tipo de flujo en que se encuentra.

\section{Método de Duong.}

Propone un modelo de declinación empírico basado en un flujo dominado por las fracturas hidráulicas en un yacimiento apretado, considera un flujo lineal con tiempo extendido. La curva de declinación que propone es:

$$
\begin{gathered}
\mathrm{q}=\mathrm{q}_{1} \mathrm{t}(\mathrm{a}, \mathrm{m})+\mathrm{q}_{\infty} \\
\mathrm{t}(\mathrm{a}, \mathrm{m})=\mathrm{t}^{-\mathrm{m}} \mathrm{e}^{\frac{\mathrm{a}}{1-\mathrm{m}}\left(\mathrm{t}^{1-\mathrm{m}}-1\right)}
\end{gathered}
$$

Donde " $m$ " es la pendiente del gráfico de $\log (\mathrm{q} / \mathrm{Np})$ vs log (tiempo en días), "a" es la intersección con el eje de las ordenadas del gráfico de log (q/Np) vs log (tiempo 
en días), “ es el caudal a 1 día de producción y también representa la pendiente de la gráfica de "q" vs "t(a,m)" y "'" es el caudal a un tiempo infinito, puede ser cero, positivo o negativo, es la intersección con el eje de las ordenadas del gráfico de "q" vs "t(a,m)".

Este método es de fácil aplicación en su uso, ya que todos sus parámetros de declinación son calculados a partir de la regresión lineal de información de producción.

\section{Método de Declinación Exponencial Extendida.}

Zhang et al proponen un método de análisis de curvas de declinación basado en un volumen de drenaje creciente (Zhang, H. E., et al, 2015).

Presentan tres parámetros de declinación. El parámetro representa la declinación temprana y brusca, producto del flujo dominante de la fractura con insuficiente soporte de presión de la matriz, inmediatamente después de la puesta en producción del pozo, el parámetro representa la declinación en tiempo tardío, representado la expansión del radio de drenaje, el parámetro $n$ es un exponente empírico.

$$
\begin{gathered}
\mathrm{q}=\mathrm{q}_{\mathrm{i}} \mathrm{e}^{-\mathrm{at}} \\
\mathrm{a}=\beta_{1}+\beta_{\mathrm{e}} \mathrm{e}^{-\mathrm{t}^{\mathrm{n}}}
\end{gathered}
$$

\section{Metodología}

\section{Análisis de pozos productores}

Se analizaron 30 pozos representativos de la cuenca Talara, productores de las formaciones apretadas: Mesa, Mogollón, Manta y Pariñas Inferior; todas en el rango de $1 \mathrm{mD}-0.01 \mathrm{mD}$, cabe resaltar que para que estos pozos produzcan volúmenes comerciales de petróleo tienen que ser estimulados mediante fractura hidráulica.

Por temas de confidencialidad se ha renombrado los pozos analizados, la Tabla 1 muestra los nombres que utilizarán los pozos en el presente artículo y sus respectivas formaciones productoras.

Tabla 1. Nombre de los 30 pozos analizados y las formaciones productoras.

\begin{tabular}{|c|c|c|c|}
\hline Pozo & $\begin{array}{c}\text { Formación } \\
\text { Productora }\end{array}$ & $\begin{array}{c}\text { Nombre de } \\
\text { Pozo }\end{array}$ & $\begin{array}{c}\text { Formación } \\
\text { Productora }\end{array}$ \\
\hline UNI 1 & Mogollón & UNI 16 & Manta \\
\hline UNI 2 & Mesa & UNI 17 & Mesa \\
\hline UNI 3 & Mesa & UNI 18 & Manta \\
\hline UNI 4 & Mesa & UNI 19 & Manta \\
\hline UNI 5 & Mesa & UNI 20 & Manta \\
\hline UNI 6 & Mesa & UNI 21 & Fm. Pariñas Inferior \\
\hline UNI 7 & Mesa & UNI 22 & Fm. Pariñas Inferior \\
\hline UNI 8 & Mesa & UNI 23 & Fm. Pariñas Inferior \\
\hline UNI 9 & Mesa & UNI 24 & Fm. Pariñas Inferior \\
\hline UNI 10 & Mesa & UNI 25 & Fm. Pariñas Inferior \\
\hline UNI 11 & Mesa & UNI 26 & Fm. Pariñas Inferior \\
\hline UNI 12 & Mesa & UNI 27 & Fm. Pariñas Inferior \\
\hline UNI 13 & Manta & UNI 28 & Fm. Pariñas Inferior \\
\hline UNI 14 & Manta & UNI 29 & Fm. Pariñas Inferior \\
\hline UNI 15 & Manta & UNI 30 & Fm. Pariñas Inferior \\
\hline
\end{tabular}

\section{Análisis determinístico}

Se han ajustado los modelos de análisis de curvas de declinación descritos anteriormente a 30 pozos seleccionados. Se ha realizado el ajuste en tres periodos, a los primeros 6, 12 y 24 meses de producción, para comparar los resultados que tienen las curvas al utilizar únicamente información en estado transitorio y cuando se combina con producción de un flujo dominado por fronteras. Además, se han comparado los modelos entre sí, seleccionando aquel que presenta mejor ajuste con la historia de producción.

Para determinar el grado de ajuste, se ha utilizado un indicador llamado "Diferencia (\%)" el cual está definido por:

$$
\text { Diferencia }(\%)=\left(\frac{\text { Volúmen acumulado }_{\text {Modelo }}-\text { Volúmen acumulado }_{\text {Real }}}{\text { Volúmen acumulado }_{\text {Real }}}\right) * 100 \%
$$

El ajuste del modelo a la historia productiva del pozo será mejor si la diferencia del cálculo de volúmenes es cercana a cero. Además, si este indicador es negativo, el ajuste estaría subestimando las reservas, caso contrario estaría sobreestimándolas.

La Figura 5 muestra un gráfico log-log donde se observa que el tiempo que le toma al pozo "UNI 1" en llegar a un flujo de depletación o dominado por fronteras es de aproximadamente 12 meses, esto indica que para poder pronosticar correctamente la producción del pozo con las ecuaciones de DCA de Arps no se debería considerar estos primeros 12 meses, sino solo la información en flujo dominado por fronteras como se describe en las consideraciones de las ecuaciones Arps. Este comportamiento se repite en los pozos restantes y 
guarda relación con lo observado en la Tabla 2, donde se muestra los parámetros de declinación del ajuste del modelo de Arps. Debido a que se utilizó información de producción en estado transitorio el parámetro $b$ sale del rango teórico y a medida que se incluye mayor información proveniente del flujo pseudo-estable se acota el parámetro $b$ a valores más coherentes.

Tabla 2. Parámetros de declinación del modelo DCA de Arps, considerando los primeros 6 meses y 24 meses de producción.

\begin{tabular}{|c|c|c|c|c|c|c|}
\hline \multirow{2}{*}{ POZO } & \multicolumn{3}{|c|}{ ARPS - 6 MESES } & \multicolumn{3}{|c|}{ ARPS - 24 MESES } \\
\hline & Qi & Di & b & Qi & Di & b \\
\hline UNI 1 & 89.1 & 4.2 & 4.1 & 89.1 & 2.7 & 2.3 \\
\hline UNI 2 & 35.2 & 3.4 & 1.8 & 35.2 & 10.7 & 3.1 \\
\hline UNI 3 & 35.6 & 4.2 & 1.7 & 35.6 & 3.9 & 1.5 \\
\hline UNI 4 & 22.5 & 2.2 & 1.9 & 22.5 & 2.3 & 3.0 \\
\hline UNI 5 & 95.8 & 3.1 & 0.2 & 95.8 & 4.8 & 1.5 \\
\hline UNI 6 & 40.7 & 63.9 & 7.7 & 40.7 & 5.5 & 2.0 \\
\hline UNI 7 & 136.9 & 12.5 & 1.3 & 136.9 & 13.7 & 1.4 \\
\hline UNI 8 & 25.1 & 17.0 & 8.0 & 25.1 & 10.8 & 2.3 \\
\hline UNI 9 & 84.2 & 4.0 & 0.2 & 84.2 & 5.6 & 0.9 \\
\hline UNI 10 & 58.4 & 22.6 & 1.3 & 58.4 & 32.5 & 1.7 \\
\hline UNI 11 & 85.5 & 24.0 & 4.6 & 85.5 & 14.4 & 2.0 \\
\hline UNI 12 & 69.0 & 6.3 & 1.2 & 69.0 & 7.0 & 1.5 \\
\hline UNI 13 & 135.0 & 24.8 & 2.8 & 135.0 & 12.9 & 2.3 \\
\hline UNI 14 & 220.6 & 5.7 & 2.1 & 220.6 & 5.4 & 1.7 \\
\hline UNI 15 & 410.0 & 11.1 & 3.7 & 410.0 & 6.3 & 2.1 \\
\hline UNI 16 & 38.6 & 2.4 & 1.6 & 38.6 & 6.0 & 6.1 \\
\hline UNI 17 & 154.6 & 15.4 & 2.0 & 154.6 & 10.0 & 1.8 \\
\hline UNI 18 & 41.5 & 0.4 & 1.0 & 41.5 & 0.5 & 4.3 \\
\hline UNI 19 & 58.5 & 5.8 & 0.7 & 58.5 & 9.6 & 1.8 \\
\hline UNI 20 & 66.3 & 15.4 & 2.9 & 66.3 & 20.3 & 3.5 \\
\hline UNI 21 & 47.9 & 16.4 & 1.8 & 47.9 & 18.1 & 1.9 \\
\hline UNI 22 & 36.0 & 40.0 & 2.3 & 36.0 & 20.0 & 3.0 \\
\hline UNI 23 & 60.8 & 2.4 & 2.0 & 60.8 & 2.5 & 1.7 \\
\hline UNI 24 & 141.2 & 3.3 & 0.9 & 141.2 & 0.9 & 1.1 \\
\hline UNI 25 & 29.3 & 2.1 & 2.8 & 29.3 & 1.4 & 1.2 \\
\hline UNI 26 & 37.7 & 1.8 & 2.0 & 37.7 & 2.1 & 2.8 \\
\hline UNI 27 & 38.7 & 25.8 & 6.0 & 38.7 & 10.2 & 3.3 \\
\hline UNI 28 & 138.2 & 12.2 & 3.5 & 138.2 & 7.8 & 2.5 \\
\hline UNI 29 & 44.4 & 23.5 & 4.3 & 44.4 & 9.2 & 3.5 \\
\hline UNI 30 & 15.7 & 4.1 & 6.7 & 15.0 & 1.2 & 3.0 \\
\hline
\end{tabular}

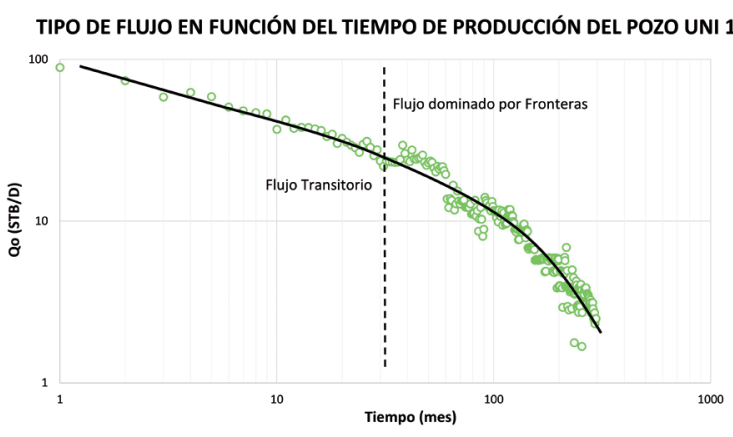

Figura 5. Tiempo que toma al pozo "UNI 1" en llegar al flujo dominado por fronteras.

En las Figuras 6 a 8 y en las Tablas 3 a 5, se muestra el procedimiento de ajuste para el pozo "UNI 1", los ajustes para los otros 29 pozos se pueden revisar en los anexos A, B y C.

Para la selección del mejor modelo de DCA, se realizó una distribución estadística de los resultados del ajuste a los 30 pozos, seleccionando aquel modelo con menor diferencia en el cálculo de reservas, además que sea conservador y con menor desviación estándar.

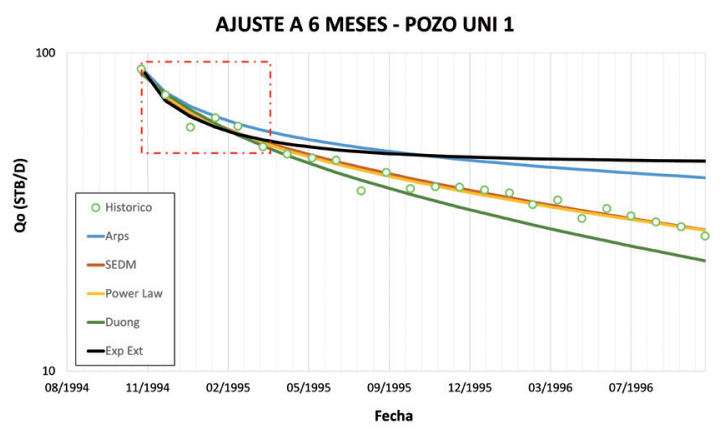

Figura 6. Ajuste de los modelos de declinación para los primeros 6 meses de producción.

Tabla 3. Resultados de ajuste con 6 meses de producciónPozo UNI 1.

\begin{tabular}{|c|c|c|}
\hline \multicolumn{2}{|c|}{ Np histórico (MSTB) } & $\mathbf{1 0 6 . 4}$ \\
\hline Método & $\begin{array}{c}\text { Reservas calculadas } \\
\text { (MSTB) }\end{array}$ & $\begin{array}{c}\text { Error } \\
\mathbf{( \% )}\end{array}$ \\
\hline Arps & 257.9 & $143 \%$ \\
\hline SEDM & 82.9 & $-22 \%$ \\
\hline Power Law & 81.5 & $-23 \%$ \\
\hline Duong & 48.6 & $-54 \%$ \\
\hline $\begin{array}{c}\text { Exponencial } \\
\text { Extendida }\end{array}$ & 228.1 & $115 \%$ \\
\hline
\end{tabular}




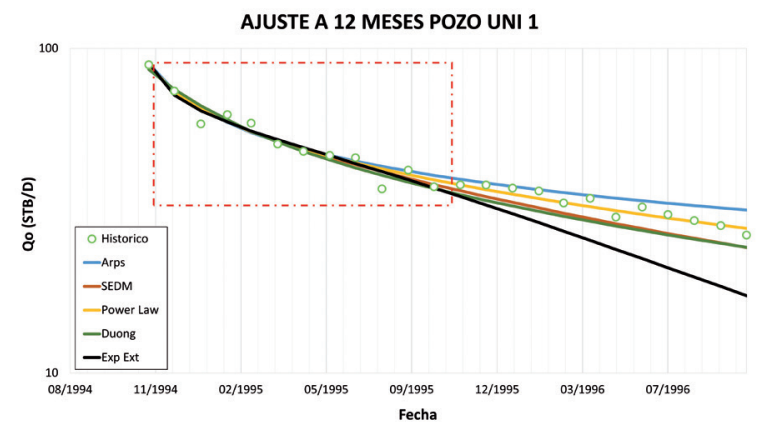

Figura 7. Ajuste de los modelos de declinación para los primeros 12 meses de producción.

Tabla 4. Resultados de ajuste con 12 meses de producción Pozo UNI 1.

\begin{tabular}{|c|c|c|}
\hline \multicolumn{2}{|c|}{ Np historico (MSTB) } & 106.4 \\
\hline Método & $\begin{array}{c}\text { Reservas calculadas } \\
\text { (MSTB) }\end{array}$ & Error (\%) \\
\hline Arps & 177.5 & $67 \%$ \\
\hline SEDM & 64.2 & $-40 \%$ \\
\hline Power Law & 87.7 & $-18 \%$ \\
\hline Duong & 68.9 & $-35 \%$ \\
\hline $\begin{array}{c}\text { Exponencial } \\
\text { Extendida }\end{array}$ & 36.5 & $-66 \%$ \\
\hline
\end{tabular}

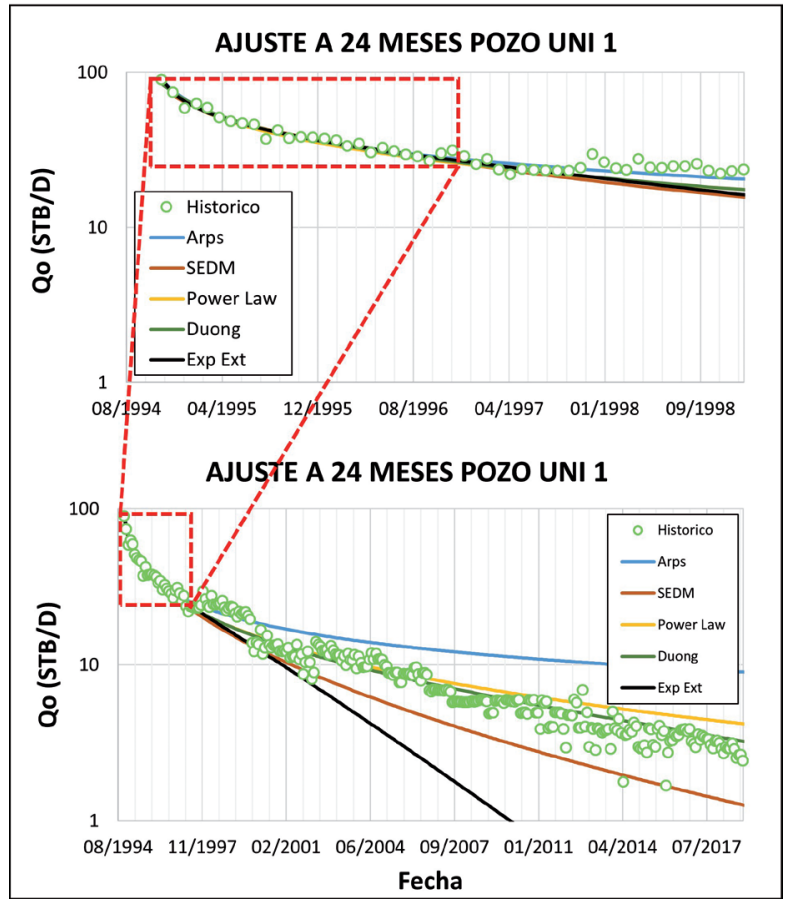

Figura 8. Ajuste de los modelos de declinación para los primeros 24 meses de producción.
Tabla 5. Resultados de ajuste con 24 meses de producción Pozo UNI 1.

\begin{tabular}{|l|c|c|}
\hline \multicolumn{2}{|c|}{ Np historico (MSTB) } & $\mathbf{1 0 6 . 4}$ \\
\hline Método & $\begin{array}{c}\text { Reservas calculadas } \\
\text { (MSTB) }\end{array}$ & $\begin{array}{c}\text { Diferencia } \\
\mathbf{( \% )}\end{array}$ \\
\hline Arps & 151.0 & $42 \%$ \\
\hline SEDM & 83.6 & $-21 \%$ \\
\hline Power Law & 103.2 & $-3 \%$ \\
\hline Duong & 103.0 & $-3 \%$ \\
\hline $\begin{array}{l}\text { Exponencial } \\
\text { Extendida }\end{array}$ & 75.4 & $-29 \%$ \\
\hline
\end{tabular}

\section{Análisis probabilístico y estimación de reservas.}

Una vez determinada la curva de mejor ajuste a los pozos analizados, en nuestro caso el modelo de Ley de Potencia, se utiliza esta para el cálculo probabilístico de reservas a futuros pozos dentro de la cuenca Talara. Dichos parámetros de declinación se muestran en la Tabla 6, además se ha creado distribuciones estadísticas con estos parámetros para definir su comportamiento e identificar la relación que tienen entre sí.

Se puede ver en las Figuras 9 - 11 que la distribución que más se ajusta a los parámetros de declinación " $n$ ", " $\hat{D}_{1}$ " y " $\mathrm{D}_{\infty}$ " es logarítmico normal, estas distribuciones se ajustaron utilizando el método de "Standard Error of the Mean" (SEM). La selección del parámetro " $\hat{q}_{1}$ " va a depender de las condiciones del pozo analizado, calculado a través de la ecuación de flujo en estado transitorio.

En la Figura 12 se puede observar la relación inversa entre los parámetros de declinación $\widehat{D}_{1} \mathrm{y}$.

Se remplaza las distribuciones de los parámetros de declinación en la ecuación 9 y utilizando la simulación Montecarlo se itera el proceso 10,000 veces para cada tiempo, obteniendo así una curva de declinación tipo probabilística.

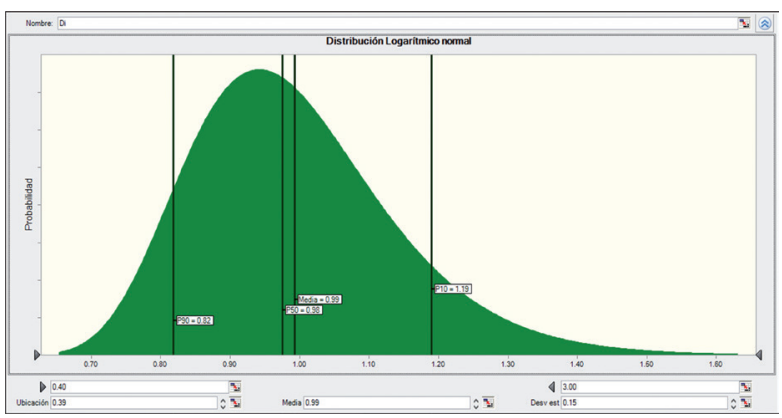

Figura 9. Distribución del parámetro. 


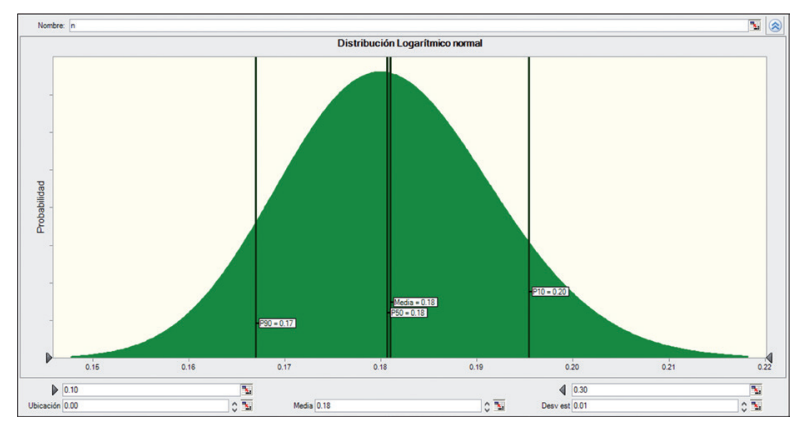

Figura 10. Distribución del parámetro $n$

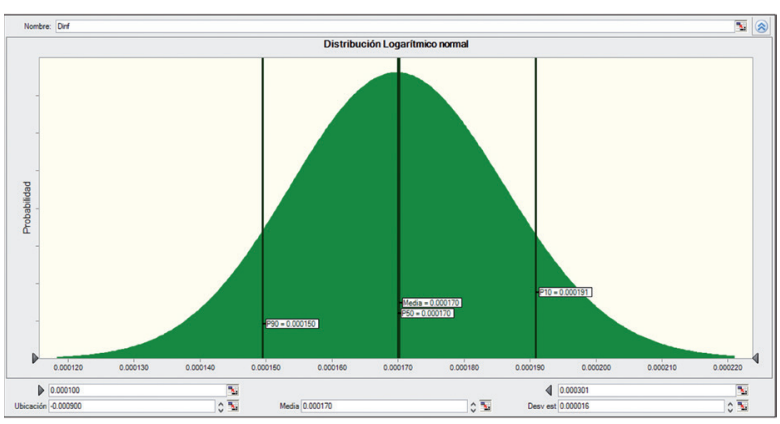

Figura 11. Distribución del parámetro

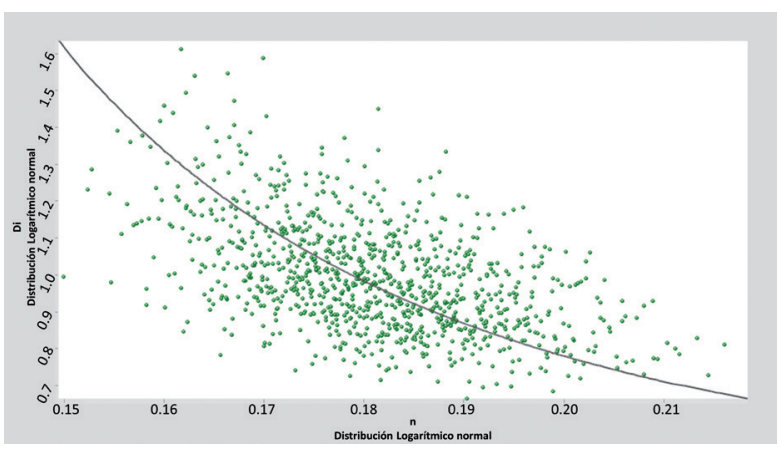

Figura 12. Relación entre los parámetros y n.

\section{Resultados y Discusión}

Los anexos A, B y C muestran los ajustes de los modelos de declinación al historial de producción de los 30 pozos, utilizando los primeros 24,12 y 6 meses de producción respectivamente; de manera descriptiva en la Tabla 7 se muestra un resumen del grado de ajuste de cada modelo de declinación, para el pozo "UNI 1".

De los anexos A, B y C se puede observar que en la mayoría de los casos el mejor ajuste, al utilizar información en estado transitorio (6 meses y 1 año), es el método de Power Law, además a medida que se utiliza mayor información la diferencia en la estimación de reservas disminuye, siendo así el mejor estimado el escenario de utilizar los 24 meses de producción. Debido a esto se continúa el análisis con este escenario.
Tabla 6. Parámetros de declinación del modelo Ley de Potencia o "Power Law".

\begin{tabular}{|c|c|c|c|c|}
\hline \multirow{2}{*}{ POZ } & \multicolumn{4}{|c|}{ Power LAW } \\
\cline { 2 - 5 } & $\mathbf{q i}^{\wedge}$ & D & & $\mathbf{n}$ \\
\hline UNI 1 & 501.3 & 0.00019 & 1.06 & 0.14 \\
\hline UNI 2 & 277.6 & 0.00010 & 1.35 & 0.14 \\
\hline UNI 3 & 200.0 & 0.00030 & 0.91 & 0.19 \\
\hline UNI 4 & 106.2 & 0.00030 & 1.08 & 0.11 \\
\hline UNI 5 & 666.7 & 0.00010 & 0.94 & 0.21 \\
\hline UNI 6 & 124.2 & 0.00010 & 0.50 & 0.26 \\
\hline UNI 7 & 1193.6 & 0.00010 & 0.91 & 0.27 \\
\hline UNI 8 & 76.9 & 0.00030 & 0.61 & 0.23 \\
\hline UNI 9 & 288.9 & 0.00030 & 0.50 & 0.30 \\
\hline UNI 10 & 673.8 & 0.00010 & 0.94 & 0.29 \\
\hline UNI 11 & 325.4 & 0.00010 & 0.50 & 0.30 \\
\hline UNI 12 & 5772.4 & 0.00010 & 2.91 & 0.12 \\
\hline UNI 13 & 162.3 & 0.00010 & 1.66 & 0.19 \\
\hline UNI 14 & 16983.4 & 0.00010 & 2.89 & 0.10 \\
\hline UNI 15 & 26092.2 & 0.00010 & 2.97 & 0.10 \\
\hline UNI 16 & 168.9 & 0.00010 & 1.11 & 0.10 \\
\hline UNI 17 & 300.0 & 0.00010 & 0.50 & 0.27 \\
\hline UNI 18 & 88.4 & 0.00010 & 0.50 & 0.11 \\
\hline UNI 19 & 70.0 & 0.00030 & 0.50 & 0.20 \\
\hline UNI 20 & 120.0 & 0.00010 & 0.68 & 0.16 \\
\hline UNI 21 & 80.0 & 0.00030 & 0.50 & 0.23 \\
\hline UNI 22 & 40.0 & 0.00010 & 0.40 & 0.20 \\
\hline UNI 23 & 140.5 & 0.00020 & 0.50 & 0.21 \\
\hline UNI 24 & 299.3 & 0.00017 & 0.50 & 0.17 \\
\hline UNI 25 & 60.0 & 0.00030 & 0.50 & 0.16 \\
\hline UNI 26 & 90.0 & 0.00010 & 0.58 & 0.16 \\
\hline UNI 27 & 110.0 & 0.00010 & 0.96 & 0.13 \\
\hline UNI 28 & 227.0 & 0.00025 & 0.50 & 0.18 \\
\hline UNI 29 & 85.0 & 0.00010 & 0.50 & 0.19 \\
\hline UNI 30 & 38.5 & 0.00020 & 0.50 & 0.15 \\
\hline
\end{tabular}

Tabla 7. Resumen del ajuste de los modelos de declinación para el pozo UNI 1

\begin{tabular}{|c|c|c|c|}
\hline \multirow{2}{*}{ Método } & \multicolumn{3}{|c|}{ Diferencia (\%) } \\
\cline { 2 - 4 } & $\mathbf{6}$ meses & $\mathbf{1 2}$ meses & $\mathbf{2 4}$ meses \\
\hline Arps & $143 \%$ & $67 \%$ & $42 \%$ \\
\hline SEDM & $-22 \%$ & $-40 \%$ & $-21 \%$ \\
\hline Power Law & $-23 \%$ & $-18 \%$ & $-3 \%$ \\
\hline Duong & $-54 \%$ & $-35 \%$ & $-3 \%$ \\
\hline Exponencial Extendida & $115 \%$ & $-66 \%$ & $-29 \%$ \\
\hline
\end{tabular}


La Tabla 8 muestra el promedio y la desviación estándar del ajuste de los modelos de declinación a los 30 pozos analizados, los valores se pueden ver en las Figuras 13-17.

Tabla 8. Distribución estadística del ajuste de los modelos de declinación utilizando los 24 primeros meses de producción.

\begin{tabular}{|c|c|c|c|c|c|}
\hline Estadística & Arps & Duong & $\begin{array}{c}\text { Exp. } \\
\text { Ext. }\end{array}$ & $\begin{array}{c}\text { Power } \\
\text { Law }\end{array}$ & SEDM \\
\hline $\begin{array}{c}\text { Prom. Diferencia } \\
(\%)\end{array}$ & $15 \%$ & $-8 \%$ & $-15 \%$ & $-12 \%$ & $-15 \%$ \\
\hline $\begin{array}{c}\text { Desviación } \\
\text { Estándar }\end{array}$ & 0.22 & 0.30 & 0.24 & 0.15 & 0.19 \\
\hline
\end{tabular}

De la Tabla 8 y la Figura 13 se puede observar que el método de Arps considerando $b>1$ sobreestima las reservas casi en todos los casos; por otro lado, se observa en las Figuras $14-17$ que los nuevos modelos de declinación son en promedio más conservadores que Arps. Sin embargo, el que presenta mayor consistencia en la estimación de reservas es el modelo de Power Law o Ley de Potencia, por ser un modelo conservador, con ajuste más cercano al valor real y el que presenta menor desviación estándar.

Cabe resaltar que el modelo de Duong realiza un buen ajuste con el historial productivo, pero en presencia de ruido suele sobreestimar las reservas, es por eso que muestra mayor desviación estándar y no es seleccionado como el mejor modelo de ajuste. Por otro lado, el modelo SEDM subestima las reservas casi en todos los casos. El modelo de exponencial extendida es el más complicado de utilizar por la relación que tienen los parámetros de declinación $\beta_{1}, \beta_{\mathrm{e}} \mathrm{y}$ n lo que genera muchas soluciones de convergencia al utilizar el método de regresión múltiple.

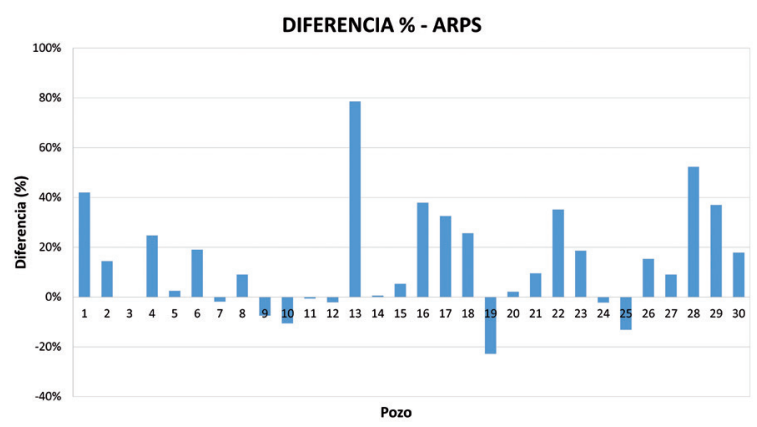

Figura 13. Diferencia porcentual de los volúmenes acumulados usando el modelo de declinación de Arps.

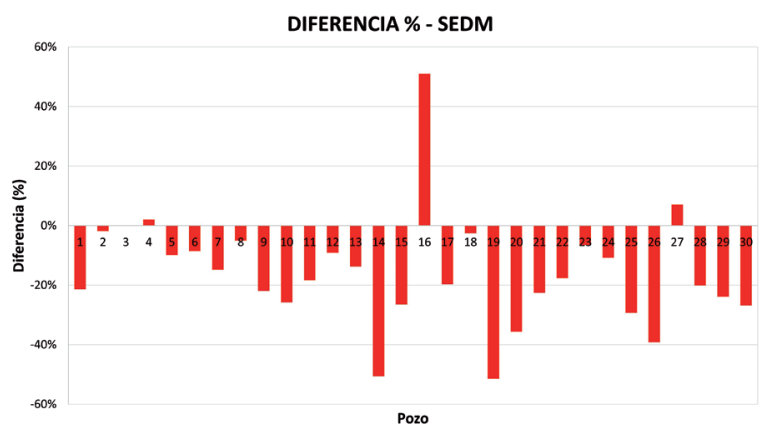

Figura 14. Diferencia porcentual de los volúmenes acumulados usando el modelo de declinación de SEDM.

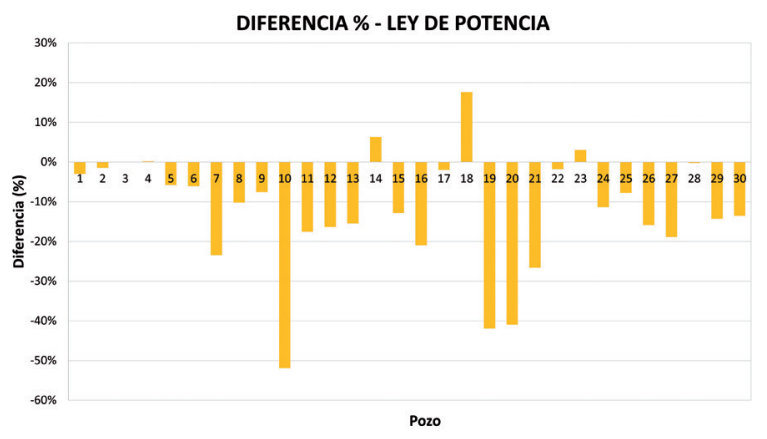

Figura 15. Diferencia porcentual de los volúmenes acumulados usando el modelo de declinación de Ley de Potencia.

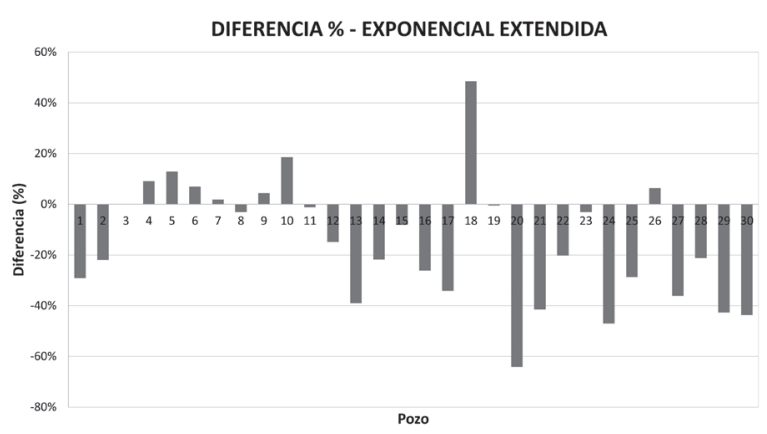

Figura 16. Diferencia porcentual de los volúmenes acumulados usando el modelo de declinación Exponencial Extendida.

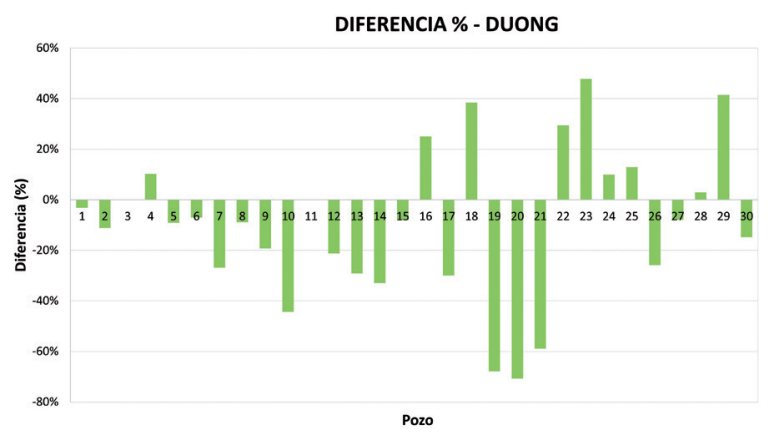

Figura 17. Diferencia porcentual de los volúmenes acumulados usando el modelo de declinación de Duong. 
En la Figura 18 se muestran las curvas de declinación tipo 1P (P90), 2P (P50) y 3P (P10) calculadas a partir de un análisis probabilístico del Método de Ley de Potencia, las cuales pueden ser usadas para la perforación de futuros pozos o trabajos de workover.

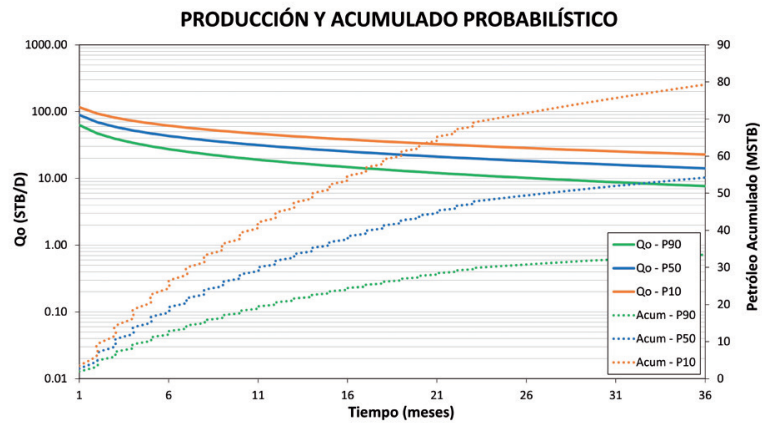

Figura 18. Curvas de declinación P10, P50 y P90.

\section{Conclusiones}

Se realizó la aplicación de los nuevos modelos de DCA para yacimientos no convencionales en los pozos productores de yacimientos apretados de la cuenca Talara. Se utilizó información de producción en estado transitorio, concluyendo que el modelo de Ley de Potencia es el más adecuado para el pronóstico de producción de estos campos.

El modelo de Ley de Potencia ajusta mejor a la historia de producción, es ligeramente conservador y presenta menor desviación estándar en los resultados, además se ha demostrado la aplicabilidad del método probabilístico en la estimación de reservas y comportamiento productivo con el modelo de Ley de Potencia.

Se identificó una relación inversa entre los parámetros "n" y " $\hat{D}_{1}$ ", la cual se utilizó en el análisis probabilístico. Además, gracias al SEM se ha obtenido un rango más acotado en los resultados de la curva de declinación. Cabe resaltar que una vez realizada la distribución de los tres parámetros de declinación " $n$ ", " $\hat{\mathrm{D}}_{1}$ " y " $\mathrm{D}_{\infty}$ " se debe ajustar el valor de " $\hat{\mathrm{q}}_{1}$ " de acuerdo al caudal inicial esperado del pozo nuevo en función de la ecuación de flujo transitorio.

Del ajuste con el historial productivo, se ha determinado que a medida que se utiliza mayor información, la diferencia en la estimación de reservas disminuye en todos los modelos de declinación. Además, se ha observado que los métodos DCA Exponencial Extendido y Duong son de difícil aplicación cuando se tiene poca información productiva.
Se recomienda la aplicación del modelo de Ley de Potencia en la estimación de reservas de pozos con poco historial productivo, pertenecientes a la cuenca Talara y productores de formaciones apretadas. Se encontró que para los pozos analizados, cuando se aplica curvas de declinación durante los primeros 24 meses de historia productiva, el método que Arps sobreestima en 15\% las reservas y presenta alta desviación estándar en sus resultados, de 0.22 , mientras que el método de Ley de Potencia ajusta con una diferencia conservadora de $-12 \%$, con desviación estándar de 0.15 .

Se recomienda seguir este artículo como una metodología de selección del mejor modelo de DCA para campos productores de formaciones apretadas o no convencionales (shale oil, shale gas).

\section{Reconocimientos}

Se agradece a la Universidad Nacional de Ingeniería por el apoyo en la elaboración de este artículo y a PERUPETRO por la información otorgada para la realización del estudio.

\section{Nomenclatura}

$\mathrm{D}_{\mathrm{i}}=$ Declinación nominal,

$\mathrm{d}=$ Constante de declinación hiperbólica, adimensional.

$\mathrm{t}=$ Tiempo, en mes para los modelos de Arps, SEDM y Exponencial Extendido y en días para los modelos de Ley de Potencia y Duong.

$\mathrm{q}_{\mathrm{i}}=$ Tasa de producción máxima (o inicial), $\mathrm{bbl} / \mathrm{d}$.

$\mathrm{n}=(\mathrm{SEDM})$ : parámetro del modelo, adimensional.

$\tau=$ Parámetro del modelo, mes.

$\widehat{\mathrm{q}}_{1}=$ Caudal "intercepto", es el caudal a 1 día de producción, bbl/d.

$\mathrm{D}_{1}=$ Constante de declinación "intercepto" a una unidad de tiempo (D a 1 día de producción), día ${ }^{-1}$.

$\mathrm{D}_{\infty}=$ Constante de declinación a un tiempo infinito, día ${ }^{-1}$.

$\mathrm{n}($ Ley de Potencia $)=$ Exponente del tiempo, adimensional.

$\hat{\mathrm{D}}_{1}=$ Constante de declinación, que es igual a $\mathrm{D}_{1} /$ n. día ${ }^{-1}$. 
$\mathrm{q}_{1}=$ Caudal a 1 día de producción, bbl/d.

$\mathrm{q}_{\infty}=$ Caudal a un tiempo infinito, puede ser cero, positivo o negativo, bbl/d.

$\mathrm{m}=$ Es la pendiente del grafico de $\log (\mathrm{q} / \mathrm{Np})$ vs $\log$ ( $\mathrm{t}$, en días), adimensional.

$\mathrm{a}($ Duong) $=$ Es la intersección con el eje $\mathrm{Y}$ del grafico de $\log (\mathrm{q} / \mathrm{Np})$ vs $\log$ ( $\mathrm{t}$, en días), adimensional.

$\beta=$ Es la constante de declinación de periodo transitorio, que debe ser más grande que .

$\beta_{1}=$ Es la constante de declinación a largos tiempos de producción,.

$\mathrm{n} \quad($ Exponencial Extendido $)=$ Exponente empírico, se recomienda que los valores estén entre 0 y 0.7 , adimensional.

\section{Referencias}

Arps, J. J. (1945). Analysis of decline curves. Transactions of the AIME, 160(01), 228-247.

Duong, A. N. (2010, January). An unconventional rate decline approach for tight and fracture-dominated gas wells. In Canadian unconventional resources and international petroleum conference. Society of Petroleum Engineers.

Fetkovich, M. J., Fetkovich, E. J., \& Fetkovich, M. D. (1996). Useful concepts for decline curve forecasting, reserve estimation, and analysis. SPE Reservoir Engineering, 11(01), 13-22.

Guardia, V. M. D., Torres, M. C., Arenas, C. E. V., Castro, R. H., Toro, G. M., \& Mendoza, O. B. (2011). Análisis de riesgo y simulación de monte carlo en la valoración de proyectosaplicación en la industria de los hidrocarburos. Fuentes, el reventón energético, 9(2).

Ilk, D., Rushing, J. A., Perego, A. D., \& Blasingame, T. A. (2008, January). Exponential vs. hyperbolic decline in tight gas sands: understanding the origin and implications for reserve estimates using Arps' decline curves. In SPE annual technical conference and exhibition. Society of Petroleum Engineers.

Páez, E. G. M., González, F. E. C., \& Duarte, C. A. M. (2016). Aplicación de series de tiempo en la realización de pronósticos de producción. Fuentes: El reventón energético, 14(1), 79-88.

Valko, P. P. (2009, January). Assigning value to stimulation in the Barnett Shale: a simultaneous analysis of 7000 plus production hystories and well completion records. In SPE hydraulic fracturing technology conference. Society of Petroleum Engineers.

Zhang, H. E., Cocco, M., Rietz, D., Cagle, A., \& Lee, J. (2015, October). An empirical extended exponential decline curve for shale reservoirs. In SPE Annual Technical Conference and Exhibition. Society of Petroleum Engineers.

Recepción: 17 de marzo de 2020

Aceptación: 11 de julio de 2020 


\section{Anexo A. Resultados del ajuste} de los modelos de DCA para los 30 pozos, utilizando los primeros 24 meses de producción

Tabla A.1. Resultados de la diferencia porcentual en el cálculo de reservas, utilizando los primeros 24 meses de producción.

\begin{tabular}{|c|c|c|c|c|c|}
\hline \multirow{2}{*}{ POZ } & \multicolumn{5}{|c|}{ Método } \\
\cline { 2 - 6 } & Arps & SEDM & $\begin{array}{c}\text { Power } \\
\text { Law }\end{array}$ & Duong & $\begin{array}{c}\text { Exponencial } \\
\text { Extendido }\end{array}$ \\
\hline UNI 1 & $42 \%$ & $-21 \%$ & $-3 \%$ & $-3 \%$ & $-29 \%$ \\
\hline UNI 2 & $14 \%$ & $-2 \%$ & $-1 \%$ & $-11 \%$ & $-22 \%$ \\
\hline UNI 3 & $-10 \%$ & $-44 \%$ & $-39 \%$ & $-29 \%$ & $-20 \%$ \\
\hline UNI 4 & $25 \%$ & $2 \%$ & $0 \%$ & $10 \%$ & $9 \%$ \\
\hline UNI 5 & $2 \%$ & $-10 \%$ & $-6 \%$ & $-9 \%$ & $13 \%$ \\
\hline UNI 6 & $19 \%$ & $-9 \%$ & $-6 \%$ & $-7 \%$ & $7 \%$ \\
\hline UNI 7 & $-2 \%$ & $-15 \%$ & $-23 \%$ & $-27 \%$ & $2 \%$ \\
\hline UNI 8 & $9 \%$ & $-5 \%$ & $-10 \%$ & $-9 \%$ & $-3 \%$ \\
\hline UNI 9 & $-7 \%$ & $-22 \%$ & $-8 \%$ & $-19 \%$ & $4 \%$ \\
\hline UNI 10 & $-11 \%$ & $-26 \%$ & $-52 \%$ & $-44 \%$ & $19 \%$ \\
\hline UNI 11 & $-1 \%$ & $-18 \%$ & $-18 \%$ & $0 \%$ & $-1 \%$ \\
\hline UNI 12 & $-2 \%$ & $-9 \%$ & $-16 \%$ & $-21 \%$ & $-15 \%$ \\
\hline UNI 13 & $79 \%$ & $-14 \%$ & $-15 \%$ & $-29 \%$ & $-39 \%$ \\
\hline UNI 14 & $1 \%$ & $-51 \%$ & $6 \%$ & $-33 \%$ & $-22 \%$ \\
\hline UNI 15 & $5 \%$ & $-26 \%$ & $-13 \%$ & $-8 \%$ & $-8 \%$ \\
\hline UNI 16 & $38 \%$ & $51 \%$ & $-21 \%$ & $25 \%$ & $-26 \%$ \\
\hline UNI 17 & $33 \%$ & $-20 \%$ & $-2 \%$ & $-30 \%$ & $-34 \%$ \\
\hline UNI 18 & $26 \%$ & $-3 \%$ & $18 \%$ & $38 \%$ & $49 \%$ \\
\hline UNI 19 & $-23 \%$ & $-51 \%$ & $-42 \%$ & $-68 \%$ & $-1 \%$ \\
\hline UNI 20 & $2 \%$ & $-36 \%$ & $-41 \%$ & $-71 \%$ & $-64 \%$ \\
\hline UNI 21 & $10 \%$ & $-23 \%$ & $-27 \%$ & $-59 \%$ & $-41 \%$ \\
\hline UNI 22 & $35 \%$ & $-18 \%$ & $-2 \%$ & $29 \%$ & $-20 \%$ \\
\hline UNI 23 & $19 \%$ & $-7 \%$ & $3 \%$ & $48 \%$ & $-3 \%$ \\
\hline UNI 24 & $-2 \%$ & $-11 \%$ & $-11 \%$ & $10 \%$ & $-47 \%$ \\
\hline UNI 25 & $-13 \%$ & $-29 \%$ & $-8 \%$ & $13 \%$ & $-29 \%$ \\
\hline UNI 26 & $15 \%$ & $-39 \%$ & $-16 \%$ & $-26 \%$ & $6 \%$ \\
\hline UNI 27 & $9 \%$ & $7 \%$ & $-19 \%$ & $-8 \%$ & $-36 \%$ \\
\hline UNI 28 & $52 \%$ & $-20 \%$ & $0 \%$ & $3 \%$ & $-21 \%$ \\
\hline UNI 29 & $37 \%$ & $-24 \%$ & $-14 \%$ & $41 \%$ & $-43 \%$ \\
\hline UNI 30 & $18 \%$ & $-27 \%$ & $-14 \%$ & $-15 \%$ & $-44 \%$ \\
\hline
\end{tabular}

Tabla A.2. Distribución estadística del ajuste de los modelos de declinación utilizando los primeros 24 meses de producción.

\begin{tabular}{|c|c|c|c|c|c|}
\hline Estadística & Arps & Duong & $\begin{array}{c}\text { Exponencial } \\
\text { Extendida }\end{array}$ & $\begin{array}{c}\text { Power } \\
\text { Law }\end{array}$ & SEDM \\
\hline Promedio & $15 \%$ & $-10 \%$ & $-15 \%$ & $-12 \%$ & $-16 \%$ \\
\hline $\begin{array}{c}\text { Desviación } \\
\text { Estándar }\end{array}$ & 0.22 & 0.30 & 0.24 & 0.15 & 0.19 \\
\hline
\end{tabular}

\section{Anexo B. Resultados del ajuste de los modelos de DCA para los 30 pozos, utilizando los primeros 12 meses de producción.}

Tabla B.1. Resultados de la diferencia porcentual en el cálculo de reservas, utilizando los primeros 12 meses de producción.

\begin{tabular}{|c|c|c|c|c|c|}
\hline \multirow{2}{*}{ Pozo } & \multicolumn{5}{|c|}{ Método } \\
\cline { 2 - 6 } & Arps & SEDM & $\begin{array}{c}\text { Power } \\
\text { Law }\end{array}$ & Duong & $\begin{array}{c}\text { Exponencial } \\
\text { Extendido }\end{array}$ \\
\hline UNI 1 & $-4 \%$ & $0 \%$ & $-23 \%$ & $-33 \%$ & $7 \%$ \\
\hline UNI 2 & $6 \%$ & $1 \%$ & $-20 \%$ & $-38 \%$ & $-5 \%$ \\
\hline UNI 3 & $1 \%$ & $7 \%$ & $-11 \%$ & $-35 \%$ & $-6 \%$ \\
\hline UNI 4 & $-3 \%$ & $5 \%$ & $19 \%$ & $12 \%$ & $6 \%$ \\
\hline UNI 5 & $-1 \%$ & $2 \%$ & $-17 \%$ & $-29 \%$ & $8 \%$ \\
\hline UNI 6 & $5 \%$ & $3 \%$ & $-6 \%$ & $-22 \%$ & $3 \%$ \\
\hline UNI 7 & $7 \%$ & $2 \%$ & $-8 \%$ & $-41 \%$ & $8 \%$ \\
\hline UNI 8 & $9 \%$ & $14 \%$ & $-11 \%$ & $-21 \%$ & $-3 \%$ \\
\hline UNI 9 & $5 \%$ & $-8 \%$ & $-7 \%$ & $-32 \%$ & $5 \%$ \\
\hline UNI 10 & $14 \%$ & $17 \%$ & $-52 \%$ & $-59 \%$ & $-35 \%$ \\
\hline UNI 11 & $-2 \%$ & $-19 \%$ & $-25 \%$ & $16 \%$ & $-33 \%$ \\
\hline UNI 12 & $-9 \%$ & $-18 \%$ & $-15 \%$ & $-39 \%$ & $-37 \%$ \\
\hline UNI 13 & $138 \%$ & $0 \%$ & $13 \%$ & $41 \%$ & $-53 \%$ \\
\hline UNI 14 & $-8 \%$ & $-76 \%$ & $-33 \%$ & $-6 \%$ & $-31 \%$ \\
\hline UNI 15 & $-17 \%$ & $-3 \%$ & $0 \%$ & $-20 \%$ & $-11 \%$ \\
\hline UNI 16 & $-18 \%$ & $-33 \%$ & $0 \%$ & $-26 \%$ & $-61 \%$ \\
\hline UNI 17 & $26 \%$ & $-22 \%$ & $-5 \%$ & $60 \%$ & $-56 \%$ \\
\hline UNI 18 & $-50 \%$ & $-60 \%$ & $-19 \%$ & $41 \%$ & $-51 \%$ \\
\hline UNI 19 & $-41 \%$ & $-32 \%$ & $-19 \%$ & $-79 \%$ & $-40 \%$ \\
\hline UNI 20 & $2 \%$ & $-36 \%$ & $-33 \%$ & $-88 \%$ & $-71 \%$ \\
\hline UNI 21 & $10 \%$ & $-13 \%$ & $-3 \%$ & $-71 \%$ & $-37 \%$ \\
\hline UNI 22 & $68 \%$ & $-37 \%$ & $10 \%$ & $201 \%$ & $-37 \%$ \\
\hline UNI 23 & $21 \%$ & $-39 \%$ & $-8 \%$ & $57 \%$ & $13 \%$ \\
\hline UNI 24 & $19 \%$ & $-22 \%$ & $8 \%$ & $78 \%$ & $-69 \%$ \\
\hline UNI 25 & $-13 \%$ & $-7 \%$ & $-1 \%$ & $46 \%$ & $6 \%$ \\
\hline UNI 26 & $-8 \%$ & $-55 \%$ & $-12 \%$ & $-38 \%$ & $-12 \%$ \\
\hline UNI 27 & $11 \%$ & $21 \%$ & $7 \%$ & $-68 \%$ & $-37 \%$ \\
\hline UNI 28 & $112 \%$ & $18 \%$ & $2 \%$ & $38 \%$ & $-41 \%$ \\
\hline UNI 29 & $149 \%$ & $106 \%$ & $13 \%$ & $39 \%$ & $-33 \%$ \\
\hline UNI 30 & $71 \%$ & $33 \%$ & $5 \%$ & $22 \%$ & $-47 \%$ \\
\hline
\end{tabular}

Tabla B.2. Distribución estadística del ajuste de los modelos de declinación utilizando los primeros 12 meses de producción.

\begin{tabular}{|c|c|c|c|c|c|}
\hline Estadística & Arps & Duong & $\begin{array}{c}\text { Exponencial } \\
\text { Extendida }\end{array}$ & $\begin{array}{c}\text { Power } \\
\text { Law }\end{array}$ & SEDM \\
\hline Promedio & $17 \%$ & $-3 \%$ & $-25 \%$ & $-8 \%$ & $-8 \%$ \\
\hline $\begin{array}{c}\text { Desviación } \\
\text { Estándar }\end{array}$ & 0.46 & 0.59 & 0.26 & 0.16 & 0.33 \\
\hline
\end{tabular}




\section{Anexo C. Resultados del ajuste} de los modelos de DCA para los

\section{0 pozos, utilizando los primeros}

\section{6 meses de producción}

Tabla C.1. Resultados de la diferencia porcentual en el cálculo de reservas, utilizando los primeros 6 meses de producción.

\begin{tabular}{|c|c|c|c|c|c|}
\hline \multirow{2}{*}{ Pozo } & \multicolumn{5}{|c|}{ Método } \\
\cline { 2 - 6 } & Arps & SEDM & $\begin{array}{c}\text { Power } \\
\text { Law }\end{array}$ & Duong & $\begin{array}{c}\text { Exponencial } \\
\text { Extendido }\end{array}$ \\
\hline UNI 1 & $125 \%$ & $-25 \%$ & $14 \%$ & $-53 \%$ & $-40 \%$ \\
\hline UNI 2 & $3 \%$ & $-4 \%$ & $-5 \%$ & $-39 \%$ & $-23 \%$ \\
\hline UNI 3 & $-1 \%$ & $6 \%$ & $14 \%$ & $-52 \%$ & $-12 \%$ \\
\hline UNI 4 & $-6 \%$ & $1 \%$ & $3 \%$ & $-8 \%$ & $9 \%$ \\
\hline UNI 5 & $-52 \%$ & $-55 \%$ & $-25 \%$ & $-43 \%$ & $-55 \%$ \\
\hline UNI 6 & $141 \%$ & $109 \%$ & $-7 \%$ & $-40 \%$ & $-34 \%$ \\
\hline UNI 7 & $-9 \%$ & $-40 \%$ & $-40 \%$ & $-57 \%$ & $5 \%$ \\
\hline UNI 8 & $83 \%$ & $70 \%$ & $-14 \%$ & $-38 \%$ & $23 \%$ \\
\hline UNI 9 & $-45 \%$ & $-48 \%$ & $-7 \%$ & $-35 \%$ & $-44 \%$ \\
\hline UNI 10 & $-27 \%$ & $-56 \%$ & $-48 \%$ & $-65 \%$ & $-28 \%$ \\
\hline UNI 11 & $119 \%$ & $46 \%$ & $-26 \%$ & $66 \%$ & $-35 \%$ \\
\hline UNI 12 & $-15 \%$ & $-21 \%$ & $-8 \%$ & $-54 \%$ & $-52 \%$ \\
\hline UNI 13 & $104 \%$ & $-12 \%$ & $15 \%$ & $94 \%$ & $-54 \%$ \\
\hline UNI 14 & $34 \%$ & $-66 \%$ & $-21 \%$ & $-35 \%$ & $-39 \%$ \\
\hline UNI 15 & $67 \%$ & $9 \%$ & $14 \%$ & $-10 \%$ & $-38 \%$ \\
\hline UNI 16 & $-59 \%$ & $46 \%$ & $-32 \%$ & $45 \%$ & $-83 \%$ \\
\hline UNI 17 & $26 \%$ & $-22 \%$ & $13 \%$ & $60 \%$ & $-56 \%$ \\
\hline UNI 18 & $-19 \%$ & $-35 \%$ & $-19 \%$ & $54 \%$ & $49 \%$ \\
\hline UNI 19 & $-75 \%$ & $-18 \%$ & $-11 \%$ & $-85 \%$ & $-51 \%$ \\
\hline UNI 20 & $-21 \%$ & $-79 \%$ & $-33 \%$ & $-93 \%$ & $-73 \%$ \\
\hline UNI 21 & $-1 \%$ & $-42 \%$ & $-3 \%$ & $-80 \%$ & $-40 \%$ \\
\hline UNI 22 & $-57 \%$ & $-63 \%$ & $-44 \%$ & $-93 \%$ & $-76 \%$ \\
\hline UNI 23 & $40 \%$ & $-22 \%$ & $15 \%$ & $73 \%$ & $22 \%$ \\
\hline UNI 24 & $-76 \%$ & $-54 \%$ & $-52 \%$ & $-52 \%$ & $-77 \%$ \\
\hline UNI 25 & $41 \%$ & $35 \%$ & $15 \%$ & $47 \%$ & $27 \%$ \\
\hline UNI 26 & $-28 \%$ & $-70 \%$ & $-20 \%$ & $-63 \%$ & $-63 \%$ \\
\hline UNI 27 & $83 \%$ & $31 \%$ & $5 \%$ & $-80 \%$ & $-63 \%$ \\
\hline UNI 28 & $111 \%$ & $39 \%$ & $10 \%$ & $-63 \%$ & $-46 \%$ \\
\hline UNI 29 & $43 \%$ & $22 \%$ & $-34 \%$ & $-71 \%$ & $-83 \%$ \\
\hline UNI 30 & $73 \%$ & $100 \%$ & $33 \%$ & $28 \%$ & $-53 \%$ \\
\hline
\end{tabular}

Tabla C.2. Distribución estadística del ajuste de los modelos de declinación utilizando los primeros 6 meses de producción.

\begin{tabular}{|c|c|c|c|c|c|}
\hline Estadística & Arps & Duong & $\begin{array}{c}\text { Exponencial } \\
\text { Extendida }\end{array}$ & $\begin{array}{c}\text { Power } \\
\text { Law }\end{array}$ & SEDM \\
\hline Promedio & $20 \%$ & $-25 \%$ & $-36 \%$ & $-10 \%$ & $-7 \%$ \\
\hline $\begin{array}{c}\text { Desviación } \\
\text { Estándar }\end{array}$ & 0.63 & 0.56 & 0.35 & 0.22 & 0.50 \\
\hline
\end{tabular}

